\title{
Design of smart home microgrid with high permeability distributed photovoltaic
}

\author{
Xiaodong $\mathrm{Cao}^{1,2}$, Shihai Yang ${ }^{1,2}$, Feng $\mathrm{Ji}^{1,2}$, Songyang Liu ${ }^{3}$ and $\mathrm{Xu} \mathrm{Zhou}^{3 *}$ \\ ${ }^{1}$ State Grid Jiangsu Electric Power Company Electric Power Research Institute, Nanjing, Jiangsu, 210000, China \\ ${ }^{2}$ State Grid Jiangsu Demand Side Management Techniques Laboratory, Nanjing, Jiangsu, 210000, China \\ ${ }^{3}$ Beijing Fibrlink Communications Co., Ltd, Fengtai District, Beijing, 100071, China
}

\begin{abstract}
The high permeability of household distributed photovoltaic is prevalent after its access. The connection between distributed generation and main network is cut off quickly and the energy storage device is used to realize energy storage. From the point of view of economy and rationality, the above-mentioned operation has become increasingly unable to meet the health of the grid after the access of distributed generation. In this paper, a construction scheme and operation strategy of household micro-grid including household energy efficiency management is designed by using equipment and equipment integration, equipment and system integration, system and system integration of household micro-grid and household energy efficiency management. It adds in-situ absorption capacity of distributed photovoltaic, realizes efficient integration of household energy efficiency management and household microgrid, effectively solves the problems of high permeability of household distributed generation, and has certain application value in the user side.
\end{abstract}

\section{Introduction}

User-side distributed photovoltaic power generation has the characteristics of adapting measures to local conditions, clean and efficient, decentralized distribution and close utilization. It can make full use of local solar energy resources, replace and reduce fossil energy consumption. It has the functions of cutting peak and filling valley, optimizing the energy structure of large power grid, and so on. It is of great significance for realizing the sustainable development of national economy. Therefore, in recent years, the government has been strongly supporting, and more and more smallcapacity distributed photovoltaics have entered the homes of ordinary people. With the increasing penetration ratio of distributed power grid in the power grid year by year, the user-side distributed power generation access presents high penetration, uncontrollable, volatility and other shortcomings increasingly prominent. Many scholars at home and abroad have studied the uncontrollability and fluctuation of home distributed power supply. Most of the domestic units and scholars adopt fast disconnection of distributed power supply and main network connection, and use energy storage devices to realize energy storage. However, the problem of high leakage rate of distributed power supply is still under study.

The high permeability of photovoltaic will cause the inverse power problem of low-voltage power grid, which will affect the security of power grid operation. Improving household photovoltaic generation absorption is a new trend and direction to solve the problem of high permeability photovoltaic. In recent years, the combination of smart grid and distributed generation has promoted the concept of household energy efficiency management. Considering the information of distributed photovoltaic capacity and based on household energy efficiency management strategy, the reasonable dispatch of household electricity load can effectively improve the absorption rate of distributed photovoltaic and enhance the stability of power grid operation. In this paper, a household microgrid system with high permeability distributed photovoltaic is designed. By combining household energy efficiency management with household microgrid, household energy management can be realized, new energy absorption rate can be increased, and the problem of distributed photovoltaic permeability can be solved.

\section{System design}

\subsection{Component structure}

Conventional household microgrid generally contains small capacity photovoltaic, energy storage system, inverters, watt-hour meters and Microgrid control units. Through the microgrid control unit, each generation unit and power unit can operate coordinately. Based on the traditional household microgrid, the user microgrid with household energy efficiency management is added with smart home (household energy efficiency management unit), energy-saving appliances and other equipment.

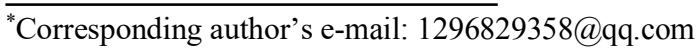


Through the effective integration of household microgrid and household energy efficiency, the household microgrid system with household energy efficiency management function is constructed.

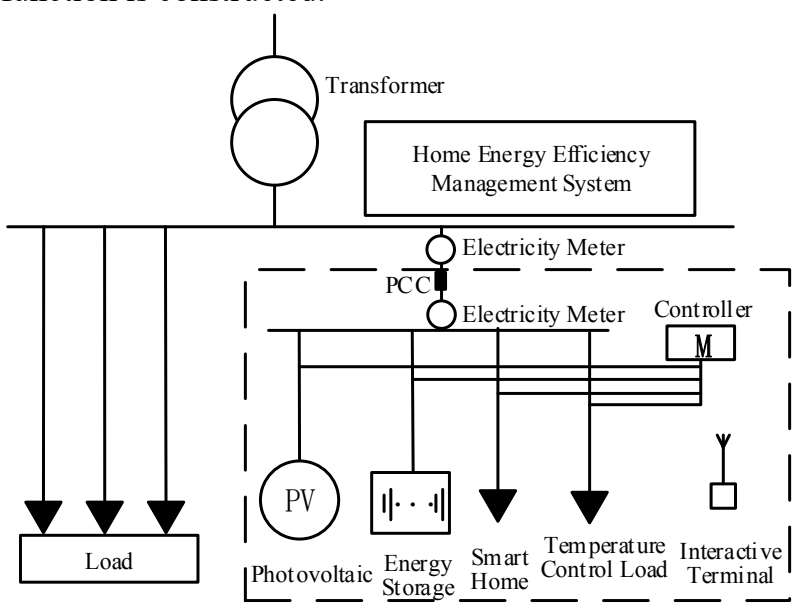

Figure 1. Household microgrid system

Intelligent household microgrid system mainly includes energy layer and information control layer. Energy layer mainly provides supply and demand services for household cold, hot and electric energy. Information control layer mainly provides safe and stable operation, energy refinement supply strategy and related comprehensive services for energy layer. In addition to conventional devices such as photovoltaic and energy storage, new energy storage media such as heat storage and cold storage are added. In addition to micro-network controller and intelligent socket, control devices such as interactive service terminals are added to the information flow transmission.

\subsection{System architecture}

The smart home microgrid system adopts standard structured design, integrates traditional energy storage system, microgrid control device and photovoltaic inverters, and uses Wi-Fi/LoRa/NB-IoT communication technology to achieve reliable communication of the whole family smart terminal equipment. The whole system includes four parts: application layer, management layer, network transmission layer and equipment support layer. Equipment support layer includes photovoltaic devices, energy storage devices, controllers, load control terminals and other underlying devices of household microgrid, as well as intelligent sockets, intelligent gateways, smart meters, interactive terminals and other devices of household energy efficiency management system. Network transmission layer uses micro-network system to communicate transmission nodes, based on LoRa/NB-IoT/WiFi and other Internet of Things wireless transmission technology, uses star networking to establish communication channels, and uploads them to the background system through the home gateway unit. The management layer includes the establishment, maintenance and configuration of network topological links, virtualization of network resources arrangement and management, condition monitoring, centralized configuration and remote upgrade of terminals and other devices. The system application layer includes power query, energy efficiency analysis, smart home, distributed power access management, home energy management and value-added services.

\section{Temperature control load control strategy}

Through the economic operation, energy saving operation, photovoltaic absorption operation, integrated operation and other target operation strategies formulated by the household energy efficiency management system with distributed generation microgrid, based on the status of household electrical equipment and indoor Multi-sensor Detection data, under the premise of guaranteeing user comfort, when the household microgrid system combines. When the network permeability is high, the household microgrid energy consumption can be realized by adjusting the time of temperature control load and the fitting technology of distributed photovoltaic/energy storage output curve.

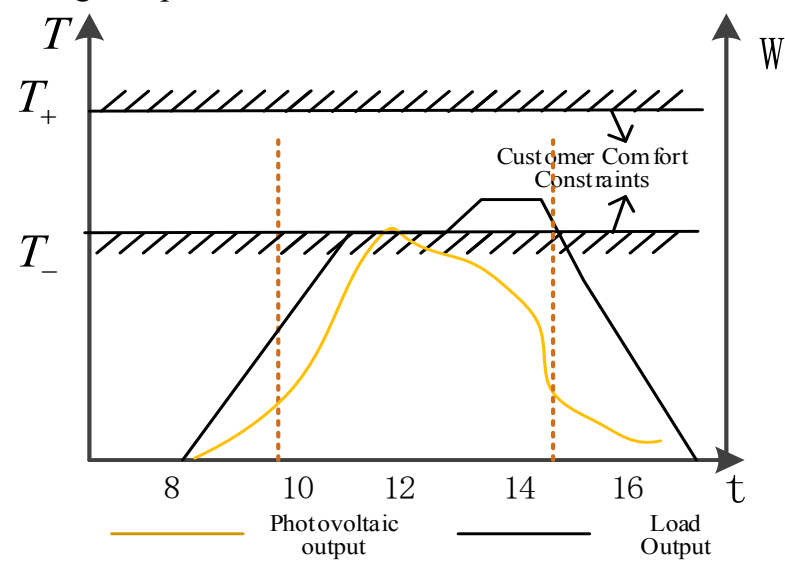

Figure 2. Temperature control load and system output fitting curve

When the grid-connected penetration rate of the household microgrid system is high/off-grid operation, the trigger state of the power system is adjusted according to the size of the system voltage offset, and the load is cut off/closed indirectly for a short time, and the load is effectively matched by the rise and fall of the voltage. There are two modes of refrigeration and heating for temperature control load. The following is a detailed description of the heating mode as an example.

\subsection{Resident Constant Temperature Load Control Method Based on Home Microgrid}

Typical household water heaters are heated mainly by utilizing the thermal conductivity resistance in the water heater of municipal electricity. The heating effect has the characteristics of a gentle rise. The energy transfer is mainly carried out by electric energy conversion, and the energy conversion balance is: 


$$
\sum_{j=1}^{2} \eta_{j} Q_{H}=Q_{p}-Q_{s a n}
$$

In the formula (1), $\mathrm{Q}_{\mathrm{H}}$ water heater consumes the heat needed by electric energy, $Q_{p}$ makes heat by electric power, $\eta_{j}$ is the heating efficiency of water heater, and $Q_{\text {san }}$ water heater loses heat when it is insulated.

In the energy conversion stage, the household microgrid water heater with distributed generators increases the output and response strategies of distributed generators, improves the energy utilization efficiency, effectively reduces the peak value of the household network, mainly through energy conversion, and in the energy conversion balance:

$$
\sum_{\mathrm{j}=1}^{4} \eta_{\mathrm{j}} \mathrm{Q}_{\mathrm{H}}=\mathrm{Q}_{\mathrm{p}}+\mathrm{Q}_{\mathrm{DG}}-\mathrm{Q}_{\mathrm{san}}-\mathrm{Q}_{\mathrm{DR}}
$$

In the formula (2), $\mathrm{Q}_{H}$ is the heat needed by the water heater to consume electricity, $Q_{p}$ is the heat generated by electricity, $Q_{D G}$ is the distributed power source to process the heat generated, $\eta_{j}$ is the heating efficiency of the water heater, the heat dissipation loss of the $\mathrm{Q}_{\mathrm{san}}$ water heater during heat preservation, and $\mathrm{Q}_{\mathrm{DR}}$ needs response to control the heat dissipation.

The interaction between tie-line power and household constant temperature load energy is realized through effective control of distributed generation in household microgrid. How to effectively increase the proportion of QDG is the key to realize the method of distributed generation absorption in household microgrid. QDG effectively monitors and manages the constant temperature load status of distributed power supply through the household energy efficiency management system with distributed power supply. In the residential constant temperature load control method of household microgrid, the residential constant temperature load control method of household microgrid is realized by detecting the integrated access voltage and frequency of household photovoltaic, monitoring and controlling photovoltaic power generation, energy storage, electricity price and constant temperature load, using coordinated optimization control strategy.

\subsection{Voltage Response Capacity of Resident Controllable Load}

On the premise of guaranteeing the user's comfort, the trigger temperature is adjusted according to the voltage offset of the whole system of the home microgrid, and the load is cut off indirectly for a short time, so as to quickly recover the voltage of the microgrid and prevent the expansion of the accident. Macroscopically, the residential thermostat controllable equipment which actively participates in the response of power grid has a uniform and random distribution of internal temperature under its normal working condition, and its trigger temperature values are slightly different. The number of devices in operation or shutdown state tends to be stable, and the power consumption is relatively stable but slightly different.

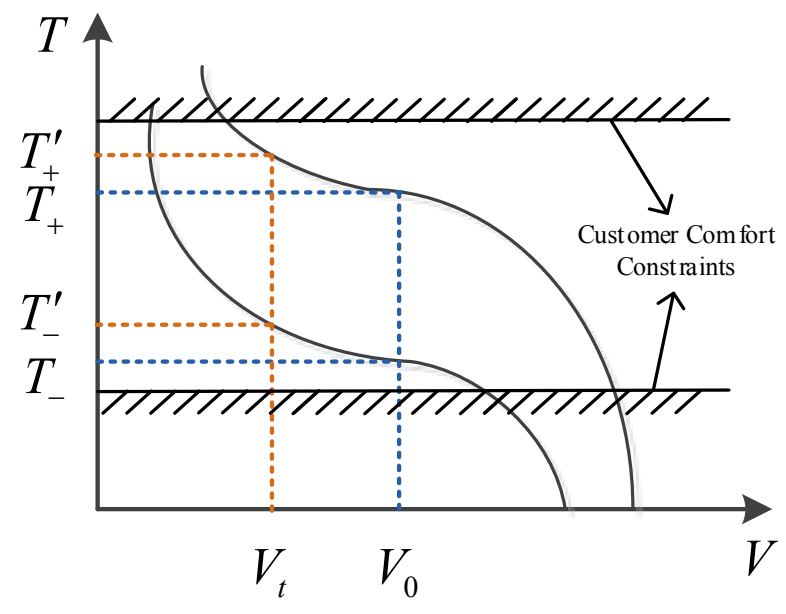

Figure 3. Temperature Load Control Method for Home Microgrid

When the temperature of the water heater is lower than the trigger temperature $\mathrm{T}$-, the water heater starts to work until the temperature of the water heater reaches the trigger temperature $\mathrm{T}+$, and stops working. Electric heaters consume electricity only during working hours, and do not consume electricity during the rest of the period Under the direct load control strategy, the trigger temperature varies with the system voltage offset. When the voltage drops, the upper and lower trigger temperatures $\mathrm{T}+, \mathrm{T}$ - of the control system move downward at the same time. On the one hand, the lowering of trigger temperature $\mathrm{T}+$ in the control system is equivalent to delaying the opening of the electric heater; on the other hand, the lowering of trigger temperature Tin the control system is equivalent to stopping the work of the heater in advance. This dual response can help the system to quickly restore to a stable state. Similarly, when the system is lightly loaded and the voltage rises, the upper and lower trigger temperatures $\mathrm{T}+, \mathrm{T}-$ of the control system move upward simultaneously, that is, to turn on the electric heater (in the switching state) ahead of time, and to delay the switching off of the heater (in the work) in order to achieve the purpose of power exchange with the household microgrid.

Through the project research, it can be concluded that temperature control can effectively respond to the fluctuation of household microgrid power. At the same time, direct load control and voltage response can be used to realize the operation control method of household microgrid based on improving the self-use efficiency of household microgrid energy, so as to realize the efficient utilization of household microgrid.

\section{Operation mode of household microgrid system}

In order to increase the uptake rate of distributed photovoltaic, the operation process of home microgrid system should include three parts: start-up, operation and stop. Through the household energy efficiency management system, the household energy orderliness can be realized, the uptake ratio of household photovoltaic 
can be increased to the greatest extent, and the electricity purchase cost of users can be reduced.

For a single household, the basic strategy of energy consumption is the same under the conditions of different photovoltaic power generation and different load demand, in order to reduce household electricity expenditure and increase photovoltaic absorption. In addition, considering other factors, the system also supports photovoltaic output tracking mode, minimum output mode, stable output mode and other operating modes.

\subsection{Photovoltaic output tracking model}

In this mode, the control load is planned according to the strategy of energy consumption, only the demand of photovoltaic output can be satisfied. Only when the demand of photovoltaic output power is lower than that of load combination power, in order to ensure that the combined load meets the constraints of operating power, the battery system can be selected for energy regulation, and priority can be followed. Renewable energy charging principle for energy storage system, when the combined operation of energy storage system demand and user load can not meet the photovoltaic output, photovoltaic surplus power grid-connected.

\subsection{Minimum output mode}

In this mode, the combined load unit should ensure the charging power of the energy storage system as much as possible. Under the condition that the non-time operation load is dispatched as little as possible, and on the basis of meeting the demand of the combined load unit, it should ensure that most of the excess photovoltaic power is stored and that less photovoltaic energy is transmitted to the grid.

\subsection{Stable output mode}

In this mode, the combined load operates at a relatively stable power level. When the distributed photovoltaic output cannot be satisfied, it can be supplemented by the discharge of the energy storage system or by utilizing the municipal electricity. When the generating power is surplus, it can be selectively charged to the energy storage system or connected to the grid. Under the stable output mode, the energy interaction with the external energy is avoided as far as possible, so that the system can run in a relatively stable state.

\section{Conclusion}

In this paper, the construction scheme of smart home microgrid with high permeability distributed photovoltaic is described in detail. A method based on the organic integration of household energy efficiency management system and Microgrid system is proposed, and the overall operation control strategy of household photovoltaic microgrid with household energy efficiency is formed. It can optimize the use of household energy, effectively increase the local absorption capacity of distributed photovoltaic, indirectly solve the problem of high permeability of distributed photovoltaic, and increase the economic benefits of residential users. It has a certain application prospect and value.

\section{Acknowledgments}

The authors greatly acknowledge the support from Nation Key Research and Development Program of China. (2016YFB0901100)

\section{References}

1. LIN Hong-yu, ZHANG Jing, XU Kun-peng, et al. Design of interactive service platform for smart power consumption [J]. Power System Technology, 2012, 36(7): 255-259.

2. ZHAO Ting, GAO Kun-lun, ZHENG Xiao-kun, et al. Research on technical framework and cyber security protection system of IOT in smart grid [J]. Electric power, 2012, 45(5): 87-90.

3. ANGEL A. AQUINO L, RAY K. A control framework for the smart grid for voltage support using agent-based technologies [J]. IEEE Trans on Smart Grid, 2011, 2(1): 161-168.

4. HAAS R, NAKICENOVIC N, AJANOVIC A, et al. Towards sustainability of energy systems: aprimer on how to apply the concept of energy services to identify necessary trends and policies [J]. Energy Policy, 2008, 36(11): 4012-4021.

5. LIANG Shi-chun, ZHANG Xiao-dong, LIN Pei-feng, et al. A Prediction Algorithm of PV Power with Hybrid Energy Storage [J]. Electric power, 2014, 47(3): 24-27.

6. LI Yong, HAN Wei, WANG Yan-hong. The Analysis and Comparison of Boiler Thermal Efficiency Correction Method Based on GB10184-88 Code [J]. Electric power, 2014, 47(3): 48-54. 